\title{
Isolation and characterization of a repeated sequence (RPS1) of Candida albicans
}

\author{
Shin-Ichi Iwaguchi, Michio Homma, ${ }^{*}$ Hiroji Chibana and Kenji Tanaka \\ Laboratory of Medical Mycology, Research Institute for Disease Mechanism and Control, \\ Nagoya University School of Medicine, Showa-ku, Nagoya 466, Japan
}

(Received 22 January 1992; revised 1 May 1992; accepted 10 June 1992)

\begin{abstract}
A repeated sequence, named RPS1, approximately $2 \mathrm{~kb}$ in size, is found mainly in chromosome 6, the second most variable chromosome among the eight chromosomes of Candida albicans. Most of the RPS1 units of chromosome 6 seem to be located within a single region of about $100 \mathrm{~kb}$ in strain FC18. In both strains FC18 and NUM812, a part of RPS1 is apparently tandemly repeated. A unit of RPS1 has been cloned and sequenced. It consists of 2114 bp and has a GC content of $40 \mathrm{~mol} \%$. The repeat unit contains smaller repeats of about 80-170 bp which are called REP1, REP2, REP3, REP4 and REP5; REP2 is duplicated. The small repeats are classified into two groups by their homology. One comprises REP1, REP2 and REP5, and the other REP3 and REP4. They are termed the REP1 and REP3 families, respectively. The two families both contain a common 29 bp sequence, called COM29. The dispersed repetitive sequence RPS1 may be involved in chromosomal rearrangements and may in part explain chromosome polymorphism in $C$. albicans. The origin of RPS1 was not determined.
\end{abstract}

\section{Introduction}

The imperfect yeast Candida albicans, which is an opportunistic pathogen, exists as a diploid in all known strains; the haploid state has not yet been found (Scherer \& Magee, 1990). C. albicans contains eight pairs of homologous chromosomes (Iwaguchi et al., 1990; Lasker et al., 1989; Wickes et al., 1991). Chromosome sizevariation has been detected in this species (Lott et al., 1987; Magee \& Magee, 1987; Merz et al., 1988; Snell et al., 1987; Iwaguchi et al., 1990). In our previous study (Iwaguchi et al., 1990), the chromosomal DNAs of $C$. albicans were resolved into 7-12 bands ranging in size from about $0.4 \mathrm{Mb}$ to about $3.0 \mathrm{Mb}$ by pulsed-field gel electrophoresis (PFGE) and no two strains showed an identical electrophoretic karyotype. Of the eight $C$. albicans chromosomes, chromosome 2 , assigned by a MGL1 probe, was more variable in size than the other

* Author for correspondence. Tel. 052-741-2111 (ext. 2116); fax 052731-9479.

Abbreviation: PFGE, pulsed-field gel electrophoresis.

The nucleotide sequence data reported in this paper have been submitted to GenBank and have been assigned the accession number M87288. chromosomes. The karyotypes of the isolates recovered from individual patients after intervals of 1-6 months were virtually identical; however, one or two chromosomes were variable in size (Asakura et al., 1991). In virtually all cases, the chromosome that varied in size was chromosome 2 . This suggested that chromosome 2 is too variable to be useful for distinguishing between strains. A similar variable chromosome was also observed by Rustchenko-Bulgac (1991) and Wickes et al. (1991) in different strains. More than $10 \%$ of clones of our strains exhibit a change in the size of chromosome 2 (Iwaguchi et al., 1992). Chromosome 2 carries an rDNA gene. The rDNA gene sequence is usually highly repeated within a single chromosomal region, in which DNA rearrangement occurs at a high frequency (Szostak $\& \mathrm{Wu}, 1980)$. We have confirmed the assumption that the clonal size-variation of chromosome 2 is derived from the rDNA cluster size-change by this type of rearrangement (Iwaguchi et al., 1992).

We found that chromosome 6 , assigned by a pTK2-9-1 probe which was prepared from a cloned DNA fragment, was the second most variable in size (Iwaguchi et al., 1990). Chromosome 6 did not always strongly hybridize to a probe which was prepared from whole chromosome 6 DNA. This difference between the fragment probe and the whole chromosome was thought to be due to the presence of a repeated sequence in 
chromosome 6 which easily translocates and results in a strong hybridization signal. Several repeated sequences have been isolated in C. albicans (Scherer \& Stevens, 1988; Sadhu et al., 1991; Soll et al., 1987; Cutler et al., 1988; Lasker et al., 1991) and were characterized as being dispersed throughout the whole genome. In the present study we report a repeated sequence which gives a hybridization profile similar to the chromosome 6 probe and which resides primarily in chromosome 6 of the strains tested.

\section{Methods}

Strains and plasmids. The strains of $C$. albicans used were $\mathrm{FC18}$, NUM46, NUM1000, NUM1039, NUM215, NUM47, NUM114, NUM961 and NUM812, whose karyotypes have been analysed previously (Iwaguchi et al., 1990).

The plasmids containing $C$. albicans genes used were pHS100 for TUB2 (Iwaguchi et al., 1990), pTK2-9-1 for pTK2-9-1 (Iwaguchi et al., 1990), and SG864 for ERG11 (Kirsch et al., 1988). The $2.2 \mathrm{~kb}$ HindIII, $7.7 \mathrm{~kb}$ EcoRI and $6.6 \mathrm{~kb}$ HindIII fragments from the respective plasmids were separated by agarose gel electrophoresis and used as probes. The probe name corresponds to the gene contained within the fragment except for pTK2-9-1. pTK2-9-1 was previously called LYS2. The gene does not complement lys 2 mutants, whereas a newly isolated DNA fragment does (Scherer \& Magee, 1990; Wickes et al., 1991), suggesting that this nomenclature was incorrect. ERG11 is the $C$. albicans gene for cytochrome P450L1Al (lanosterol 14 alphademethylase).

The C. albicans repeat sequences used were 27A (Scherer \& Stevens, 1988), Ca3 (Sadhu et al., 1991), Ca7 (Soll et al., 1987) and the MspI fragment (Cutler et al., 1988).

Preparation of yeast chromosome DNA for PFGE. The sample plug containing yeast chromosome DNA for PFGE was prepared as described previously (Iwaguchi et al., 1990).

Pulsed-field gel electrophoresis ( $P F G E$ ). PFGE was carried out by the CHEF method (Chu et al., 1986) using the Pulsarphor system with a hexagonal electrode array (Pharmacia-LKB). The yeast chromosome DNAs were separated in a $0.8 \%$ agarose gel usually with a $300 \mathrm{~s} \mathrm{switch}$ at $140 \mathrm{~V}$ for $24 \mathrm{~h}$ followed by a $1200 \mathrm{~s}$ switch at $80 \mathrm{~V}$ for $48 \mathrm{~h}$ as described previously (Iwaguchi et al., 1990). Chromosomes of Saccharomyces cerevisiae (X2180-1A; Mortimer \& Schild, 1985), chromosomes of Schizosaccharomyces pombe (HM422 derived from strain 972h-; Fan et al., 1988), $\lambda$ concatemer ladder DNA (Bio-Rad) and $\lambda$ DNA digested by EcoT14I (TAKARA Co.) were used as the molecular size reference markers.

Preparation of chromosome 6 probe for Southern hybridization. The chromosome 6 probe was prepared as described previously (Iwaguchi et al., 1992) following separation of chromosomal DNAs by PFGE in a $0.7 \%$ agarose gel.

Preparation of genomic DNA. Yeast strains were grown at $30^{\circ} \mathrm{C}$ in $50 \mathrm{ml}$ YPD broth and early stationary phase cells were collected and washed once with $30 \mathrm{ml} 50 \mathrm{~mm}$-sodium EDTA ( $\mathrm{pH} \mathrm{7.5)} \mathrm{containing}$ $0 \cdot 3 \mathrm{ml} \mathrm{2-mercaptoethanol.} \mathrm{After} \mathrm{centrifugation,} \mathrm{the} \mathrm{cell} \mathrm{pellets} \mathrm{were}$ suspended in $1 \mathrm{ml} 50 \mathrm{~mm}$-sodium citrate buffer ( $\mathrm{pH} \mathrm{5.8)} \mathrm{containing}$

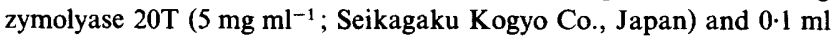
2-mercaptoethanol. After $30 \mathrm{~min}$ incubation at $37^{\circ} \mathrm{C}, 0.5 \mathrm{ml}$ proteinase $\mathrm{K}$ solution $\left(2 \mathrm{mg} \mathrm{ml}^{-1}\right)$ and $2 \mathrm{ml} 10 \%$ sodium dodecyl sulphate were mixed with the suspension and the mixture was further incubated for
$2 \mathrm{~h}$ at $37^{\circ} \mathrm{C}$ and then for $30 \mathrm{~min}$ at $55^{\circ} \mathrm{C}$. The lysate was extracted twice with an equal volume of phenol/chloroform $(1: 1, \mathrm{v} / \mathrm{v})$ and once with an equal volume of chloroform/isoamyl alcohol $(24: 1, v / v)$; the aqueous phases were mixed with 2 vols $99 \%(v / v)$ ethanol. After storage at $-20{ }^{\circ} \mathrm{C}$ overnight, the precipitates were removed and washed once with $70 \%$ ethanol. Dried precipitates were dissolved in $8 \mathrm{ml} \mathrm{TE}(10 \mathrm{mM}$-Tris/ $/ \mathrm{HCl}, 1 \mathrm{mM}-\mathrm{EDTA}, \mathrm{pH} 8.0)$ and stored at $4{ }^{\circ} \mathrm{C}$.

Southern hybridizations. Southern hybridizations were performed as described previously (Iwaguchi et al., 1990) using C. albicans cloned fragments of plasmids and chromosome 6 of strain FC18 prepared by the method described above.

The intensity of hybridization signal with the probes was measured with the BAS2000 image analyser system (Fuji Film Co.). The signal intensity of the TUB2 probe was used as a reference for a single-copy gene.

XhoI digestion of total chromosomal DNA in PFGE sample plugs. Sample plugs for PFGE were equilibrated in restriction buffer $(200 \mu \mathrm{l})$ for $30 \mathrm{~min}$ at room temperature. The plugs were then transferred to $200 \mu$ of fresh restriction buffer containing 20 units of XhoI and incubated overnight at $37^{\circ} \mathrm{C}$. After the sample plugs had been washed once with 50 mM-EDTA (pH 9.0), the digested chromosomal DNA fragments in the plugs were separated by PFGE.

Restriction analysis of chromosomes separated by PFGE. Chromosomes were separated in a $0.7 \%$ agarose gel by PFGE, stained with ethidium bromide, and destained with distilled water. The chromosome bands were cut out with a razor blade under illumination by $365 \mathrm{~nm}$ UV light. Ethidium bromide was immediately extracted from the gel slice with three $10 \mathrm{~min}$ washes with 2-butanol saturated with $1 \mathrm{~m}-\mathrm{NaCl}, 1 \mathrm{~mm}$-EDTA, $10 \mathrm{~mm}$-Tris/ $\mathrm{HCl}(\mathrm{pH} \mathrm{8} \cdot 0)$. The gel slices were then equilibrated in $200 \mu \mathrm{l}$ of restriction buffer and transferred into $200 \mu \mathrm{l}$ of fresh restriction buffer containing 20 units of $S m a \mathrm{I}$ or $\mathrm{XhoI}$ and incubated overnight at $30^{\circ} \mathrm{C}$ and $37^{\circ} \mathrm{C}$, respectively.

For partial digestion of chromosome 6 by SmaI, the enzyme concentration was serially twofold diluted from 10 units per $200 \mu \mathrm{l}$ restriction buffer. The enzyme reaction was carried out for $4 \mathrm{~h}$ at $30^{\circ} \mathrm{C}$.

Cloning of the repeated sequence (RPSI). Genomic DNA of strain NUM812 was digested with EcoRI, and the DNA fragments were ligated with EcoRI-digested plasmid pUC18. The ligated DNA was transformed into Escherichia coli JM109. Plasmid DNA from each transformant was digested with $E c o$ RI; the size of inserted fragments was checked by agarose gel electrophoresis. A plasmid containing a fragment of $2 \cdot 1 \mathrm{~kb}$ was selected for further analysis.

DNA sequence. The DNA fragment was cloned into M13mp18 in both orientations. Sequence analysis was performed by the dideoxynucleotide method, with the modified T7 DNA polymerase sequence kit (US Biochemical Corp.) and with the $B c a$ polymerase sequence kit (TAKARA Co.). A sequential series of deletions of these clones was prepared by means of a Kilo-Sequence deletion kit (TAKARA Co.). Part of the region was sequenced by using special synthesized primers. We sequenced overlapping clones in both orientations.

Sequence data manipulations and analyses were carried out by the software package, DNASIS (TAKARA Co.).

\section{Results}

\section{Hybridization profile obtained by using a chromosome 6 probe}

The whole of chromosome 6 from strain FC18 was used to probe chromosomal DNA of nine C. albicans strains 

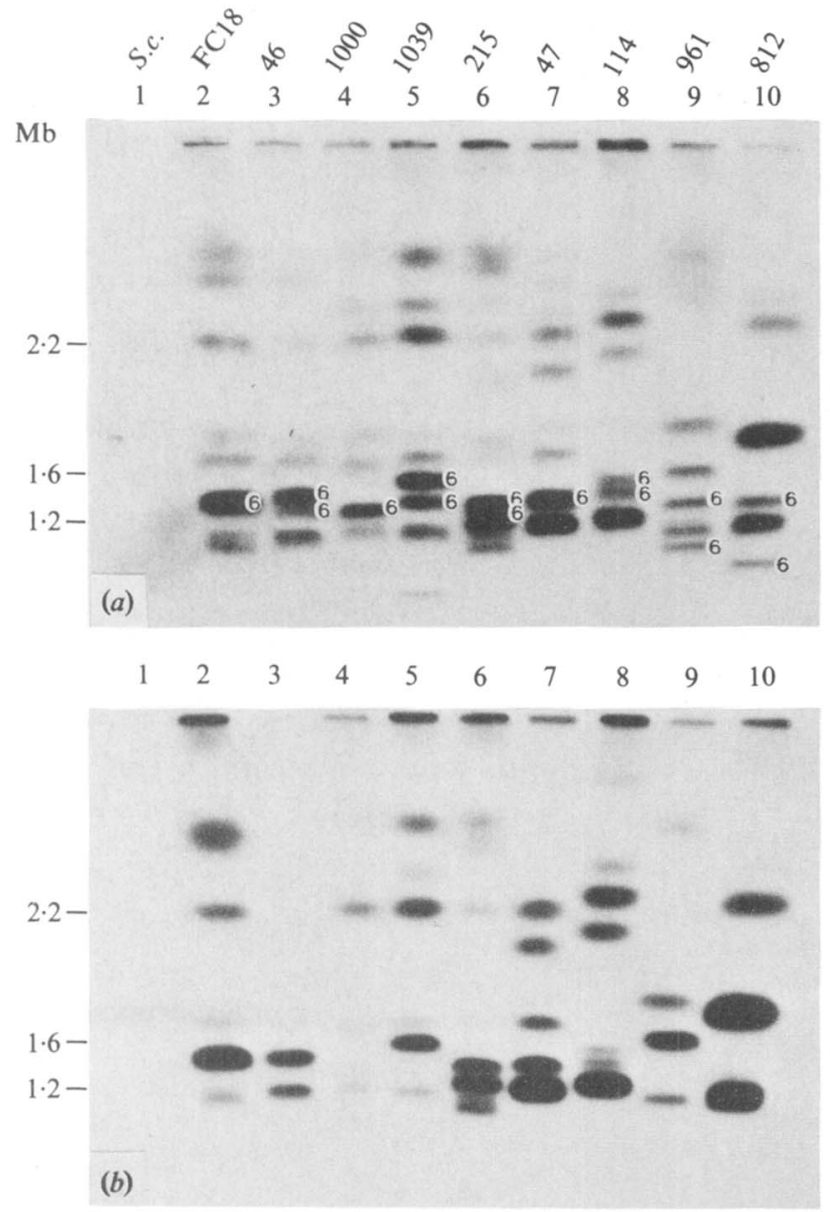

Fig. 1. Southern hybridization profiles obtained by using the FC18 chromosome 6 DNA probe $(a)$ and the RPSl probe $(b)$. The chromosomal DNAs were separated using a $300 \mathrm{~s}$ switch time at $140 \mathrm{~V}$ for $24 \mathrm{~h}$ followed by a $1200 \mathrm{~s}$ switch time at $80 \mathrm{~V}$ for $48 \mathrm{~h}$. The chromosomes were prepared from C. albicans FC18 (lane 2), NUM46 (lane 3), NUM1000 (lane 4), NUM1039 (lane 5), NUM215 (lane 6), NUM47 (lane 7), NUM114 (lane 8), NUM961 (lane 9), NUM812 (lane 10) and from $S$. cerevisiae $X 2180-1 A$ (lane 1). The numbers on the left indicate the size of chromosomal DNAs from $S$. cerevisiae. In $(a)$, the number 6 on the figure indicates the bands assigned by a chromosome 6 marker probe, pTK2-9-1 or ERG11

(Fig. $1 a$ ), whose electrophoretic karyotype had been previously analysed and for which each chromosome band had been assigned by eight cloned probes (Iwaguchi et al., 1990). In three strains (FC18, NUM1000 and NUM1039), the chromosome 6 probe hybridized to the same chromosome band as assigned by pTK 2-9-1 and ERG11 probes. These probes are cloned DNAs used as specific marker probes for chromosome 6 (data not shown). In several other strains, the FC18 chromosome 6 probe did not primarily hybridize to the band corresponding to chromosome 6 as determined by using the specific marker probes ERG11 and pTK2-9-1, or it strongly hybridized to other chromosome bands. To

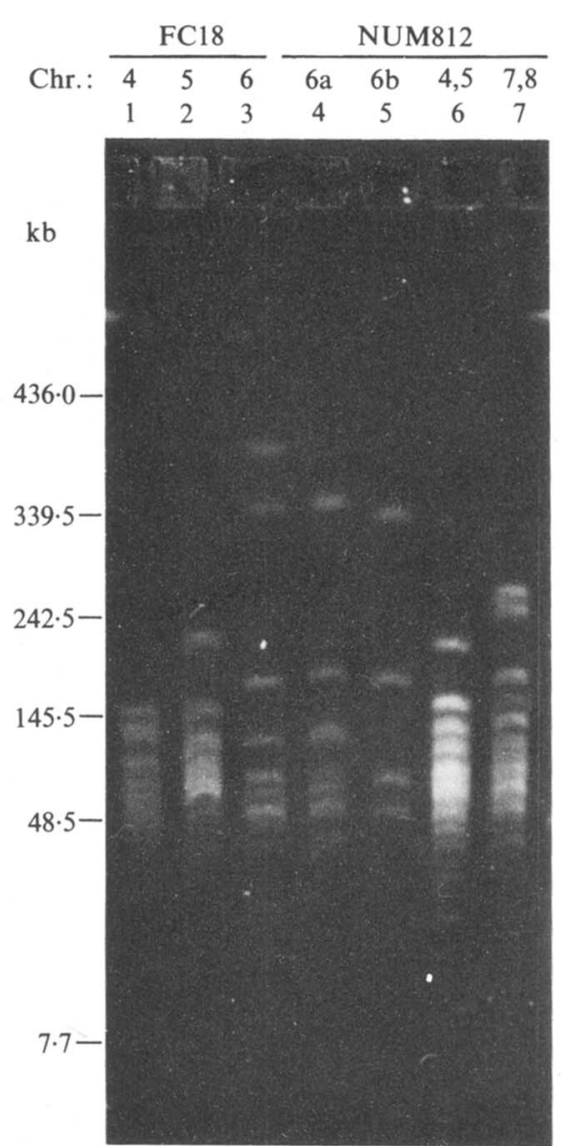

Fig. 2. SmaI digestion profiles of chromosomes. Bands containing either chromosome 4,5 or 6 of $\mathrm{FC} 18$, or chromosome 4 and $5,6 \mathrm{a}, 6 \mathrm{~b}$, or 7 and 8 of strain NUM812 were cut out from a PFGE gel. Each of the chromosome bands was treated with $S m a I$ and separated by PFGE using a linear ramping switch time from $20 \mathrm{~s}$ to $40 \mathrm{~s}$ at $200 \mathrm{~V}$ for $18 \mathrm{~h}$ in a $1 \%$ agarose gel. Lanes 1 to 3 show the digestion profiles of chromosome 4, 5 and 6, respectively, of strain FC18. Lanes 4 and 5 show the profiles of chromosome 6 of NUM812, whose homologues were separated, the larger being referred to as ' $a$ ' and the smaller as ' $b$ '. Chromosomes 4 and 5, or 7 and 8, were not separated in NUM812, so the mixed-band profiles are shown in lanes 6 and 7 . Numbers on the left indicate the size markers of $\lambda$ ladder concatemer and $\lambda$ DNA digested by EcoT14I.

clarify this apparent contradiction, we investigated one case in more detail. Because the FC18 chromosome 6 was recognizing bands corresponding to NUM812 chromosomes $4 / 5$ and $7 / 8$, we isolated these bands and NUM812 chromosome 6 bands and subjected them to restriction digest analysis using SmaI in an attempt to identify restriction fragment polymorphisms (Fig. 2). The digestion pattern of chromosome 6 of FC18 was similar, although not identical, to that of chromosome 6 of NUM812, which very weakly hybridized to the FC18 chromosome 6 probe. However, the strongly hybridizing bands (containing chromosome 4 and 5, or chromosome 7 and 8) of NUM812 did not give similar patterns to that 


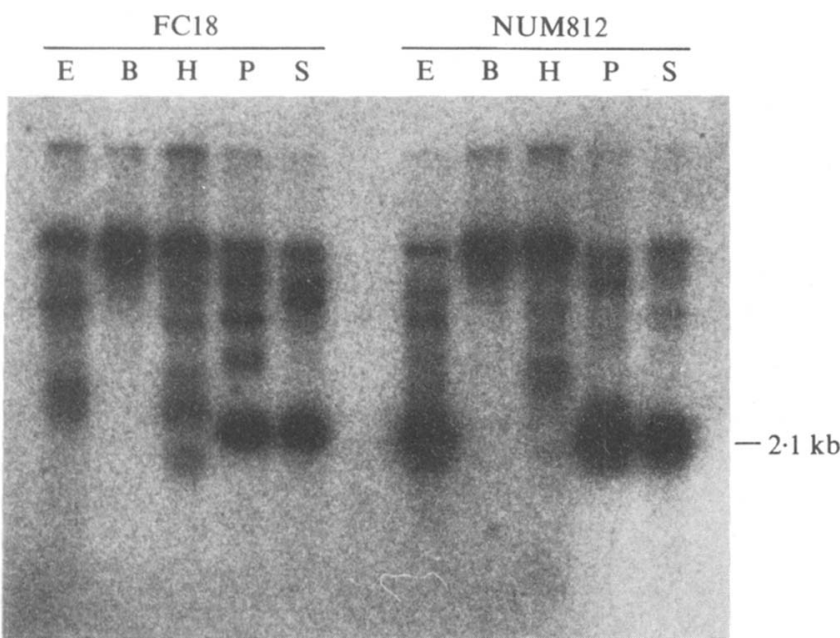

Fig. 3. Hybridization profile of a chromosome 6 probe with genomic DNA of FC18 and NUM812. The FC18 chromosome 6 probe was hybridized to total genomic DNA digested with $\operatorname{EcoRI}(\mathrm{E}), \operatorname{BamHI}(\mathrm{B})$, HindIII (H), PstI (P) or SmaI (S). of chromosome 6 (Fig. 2, lanes 6 and 7). This suggests that the FC18 chromosome 6 probe recognized other chromosomes whilst the cloned specific marker probes were more useful for chromosome assignment in this case.

The intensity of the FC18 chromosome 6 hybridization signal was very strong and was similar to that obtained when using chromosome 2 , which carries a highly repeated rDNA gene cluster, as a probe for PFGE blots (Asakura et al., 1991; Iwaguchi et al., 1992). From the above lines of evidence, it is inferred that chromosome 6 of FC18 contains a sequence which frequently translocates, resulting in strong signals on other chromosomes. This feature suggests the presence of a repeated sequence or a transposable element.

\section{Cloning of a $2.1 \mathrm{~kb}$ repeated fragment (RPS1)}

When the FC18 chromosome 6 probe was hybridized to total genomic DNA digested by various restriction
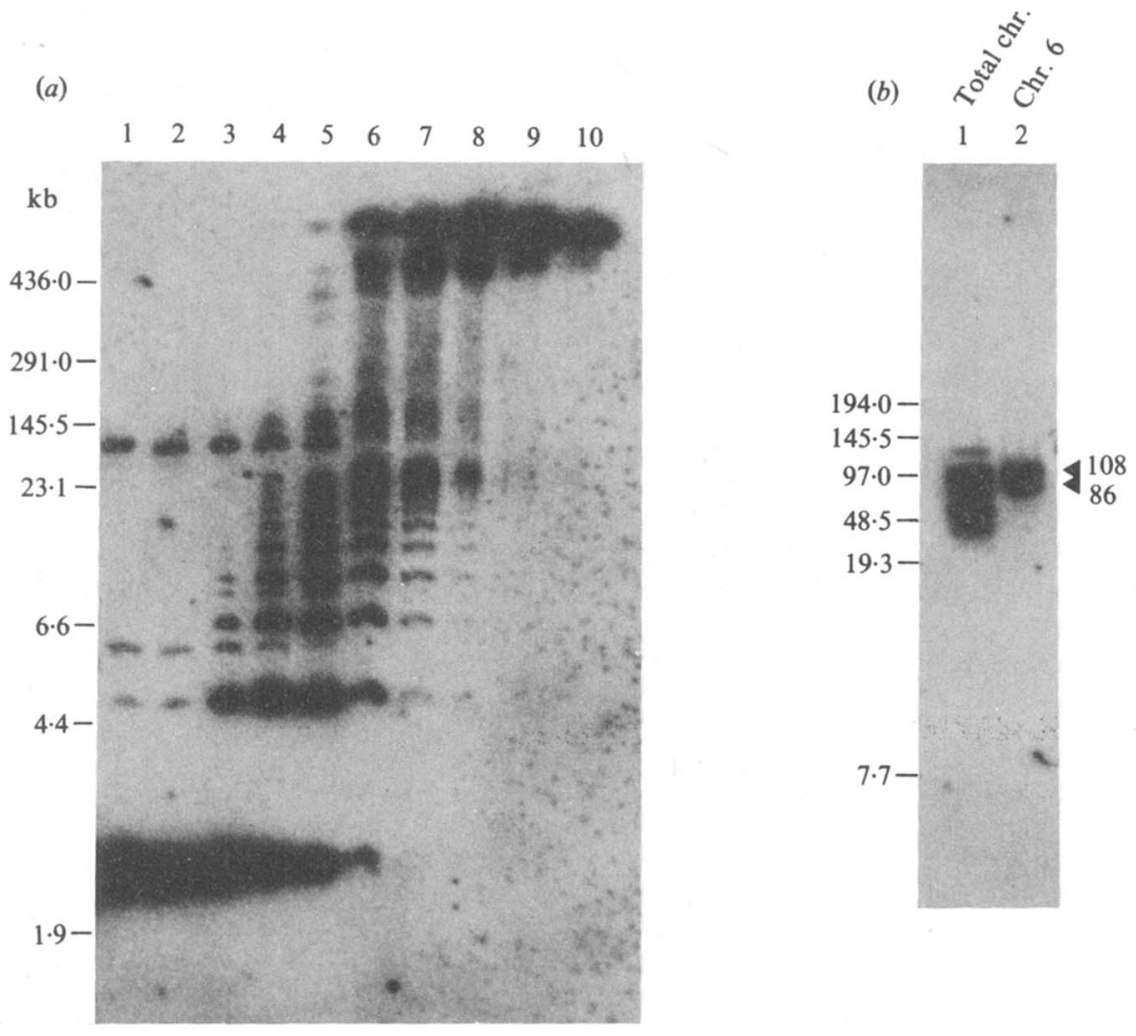

Fig. 4. Distribution of RPS1 in chromosome 6. (a) Partial digestion profiles of chromosome 6 by SmaI. The FC18 chromosome 6 band was cut out from a PFGE gel and partially digested by SmaI. The enzyme concentration was serially diluted twofold from 1 to 10 . After PFGE using a linear ramping switch time from $5 \mathrm{~s}$ to $25 \mathrm{~s}$ at $200 \mathrm{~V}$ for $10 \mathrm{~h}$ in a $1 \%$ agarose gel, Southern hybridization using the RPS1 probe was carried out. (b) XhoI fragments containing RPS1. PFGE sample plugs (Total chr.) and excised chromosome 6 band (Chr. 6 ) were treated with $X$ hoI and separated by PFGE using a linear ramping switch time from $10 \mathrm{~s}$ to $40 \mathrm{~s}$ at $200 \mathrm{~V}$ for $15 \mathrm{~h}$ in a $1 \%$ agarose gel. After PFGE, Southern hybridization using a RPS1 probe was performed. The arrowheads show two fragments $(86 \mathrm{and} 108 \mathrm{~kb})$ detected in $X h o I$ digests of the chromosome 6 band. The numbers on the left of $(a)$ and $(b)$ indicate the size markers of $\lambda$ concatemer ladder and $\lambda$ DNA digested by EcoT14I or HindIII. 
enzymes (EcoRI, BamHI, HindIII, Pst I and SmaI), it strongly hybridized to a $2 \cdot 1 \mathrm{~kb}$ fragment after $P$ st $\mathrm{I}$ and SmaI digestion of FCl8, and EcoRI, PstI and SmaI digestion of NUM812 genomic DNA (Fig. 3). This suggests that chromosome 6 of FC18 contains a $2 \cdot 1 \mathrm{~kb}$ repeated sequence.

To clone the $2 \cdot 1 \mathrm{~kb}$ fragment, genomic NUM812 DNA was digested by EcoRI and fragments were cloned into the EcoRI site of pUC18. The insert sizes were checked by EcoRI digestion, and a plasmid, pSI3-12, containing a $2 \cdot 1 \mathrm{~kb}$ fragment was obtained. This fragment probe gave similar hybridization profiles as the FC1 8 chromosome 6 probe (Fig. 1 b). This suggested that the high-intensity signals obtained when using the FC18 chromosome 6 probe were mainly derived from the $2.1 \mathrm{~kb}$ repeated fragment. This cloned $2.1 \mathrm{~kb}$ fragment was named RPS1.

Although the RPS1 probe strongly hybridized to the chromosome bands usually assigned by pTK2-9-1 or ERG11, it also weakly hybridized to almost all of the other chromosomes (Fig. 1b). In strains NUM215, NUM47, NUM114 and NUM812, some chromosomes other than chromosome 6 strongly hybridized to the RPS1 probe. It is worth noting that only chromosome 4, as assigned by the ADE2 probe, did not hybridize to the RPS1 probe in any of the strains tested.

\section{Distribution of the RPSI sequence on the chromosome}

RPSI contains unique PstI and SmaI sites. When total genomic DNA was digested by either of these two enzymes, a fragment of the same size as RPS1 was detected (Fig. 3). This strongly suggested that some RPS1 sequences were tandemly repeated. To confirm this, chromosome 6 of FC18, which was prepared by cutting out chromosome 6 separated on PFGE, was partially digested by SmaI and hybridized to the RPSI probe (Fig. 4). The RPS1 repeat unit was strongly detected in the completely digested sample (Fig. $4 a$, lane $1)$, and the fragments with sizes corresponding to multiples of the RPS1 unit were detected as a ladder of bands in partially digested samples (Fig. 4a, lanes 3-7). The ladder seems to consist of at least ten bands, suggesting that a minimum of 10 RPSI units constitute a tandemly repeated cluster.

The intensity of the RPS1 hybridizing signal in SmaI fragments of total chromosomal DNA in sample plugs of strains FC18 and NUM812 was measured by the BAS2000 image analyser system. The RPS1 signal of FC18 and NUM812 was about 30 and about 40 times, respectively, stronger than that of the TUB2 signal in the same Smal-digested sample. Using the signal of the TUB2 probe, whose GC content is similar to that of the RPS1 probe (see below), as a reference example for a

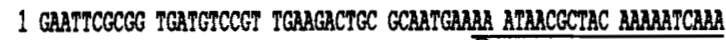

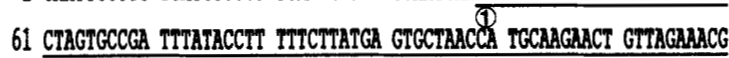

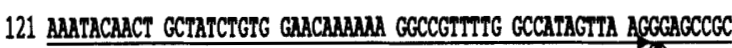

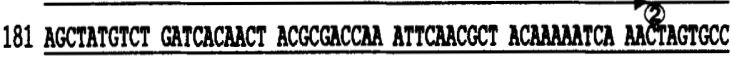

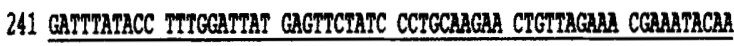

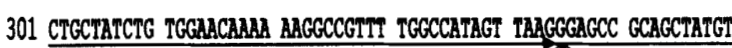

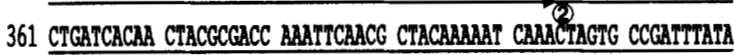

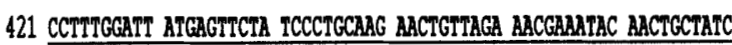

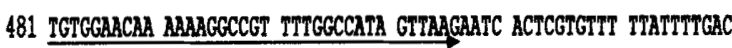
541 TACTCCTCGA CCAARAGAGG CGTTACAAA ACCAAACTA IACCGTTTY AACCCAGGAC

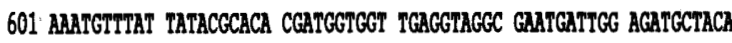

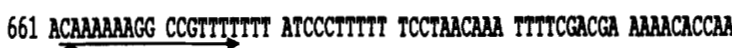

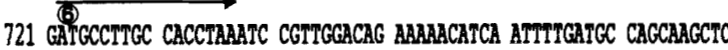
781 ATTCAAGCAT CAATTTCAAG CTACATGTT GCGCASCAMA CCACTAGATT TTCAAACAR

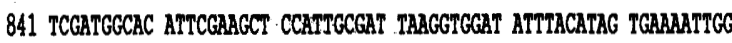

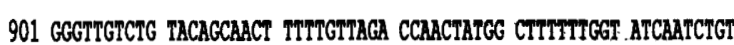

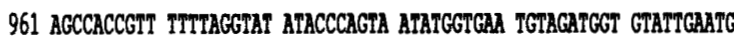

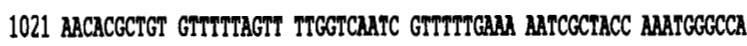

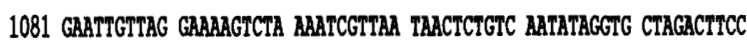
1141 CAAGGTCGG TCACTTRAA GGCCCATTTT GTRACCAACT GTCAMCTRA GTTTGGTCGA 1201 AGGAGTCCA ATATTCGTGG AGATATPGTC AAAAACCAA TATTTCAAG TTCCATCCA

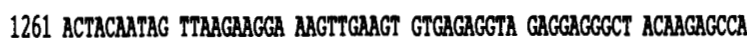
1321 ATCAGGTCCC GATTTGTGCC CIACAGTTGT GGTTTRAAM CCCGAGACAA TCGTTAATA 1381 CGGTAATTAG TTGGGTCCTG CAGGAGCAAA MAGGCCGTT TGICCARAGT TABGAGCACC

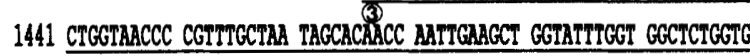

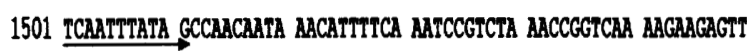

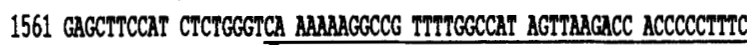

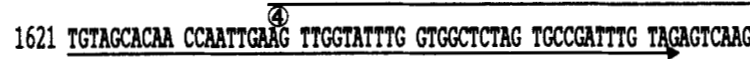

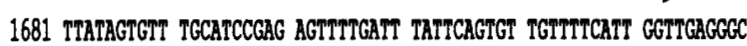

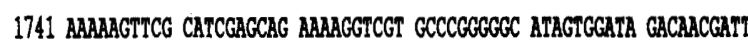

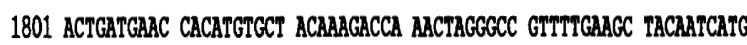

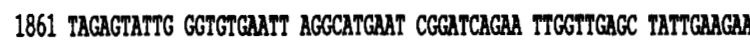
1921 AaCGTTTTCT CCGTCGAAMT GTCAAATPA CTCCCCCAAG CCTGACACAG TCACTTTCGA 1981 TGCTAGAAG ACCCAACTAG TGCCAGTTCA TAGCCATASG ATGTAGTAC TARCACTGTA

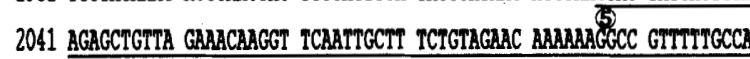

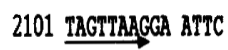

Fig. 5. Nucleotide sequence of RPS1. The sequence of RPS1 is $2114 \mathrm{bp}$ long. The repeat sequences within it, namely REP1 (39-172, $134 \mathrm{bp),}$ REP2 (173-344 and 345-516, 172 bp), REP3 (1407-1511, 105 bp), REP4 (1579-1673, 95 bp), REP5 (2027-2108, 82 bp) and REP6 (662$677,16 \mathrm{bp}$ ), are underlined, with the corresponding number circled.

gene present in a single copy per haploid genome, it was inferred that about 60 and about 80 units of RPS1 were contained in single FC18 and NUM812 cells, respectively.

When chromosome 6 DNA of FC18 was digested by 
(a)

REP2 GGAGCCGCAGCTATGTCTGATCACAACTACGCGACCAAATTCAACGCTACAAAAATCAAA REP1

TTATACCTTTGGATTATGAGTTCTATCCCTGCAAGAACTGTTAGAAAC

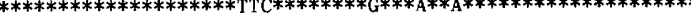
$* * \mathrm{~A} * * * * \mathrm{GA} * * * \mathrm{~T} * * * * \mathrm{G} * * * * * * * * * * *$

COM29

REP2 AAA-TACAACTGCTATCTGTGGAACAAAAAAGGCCGTTTTGGCCATAGTTAA

REP1 $* * *-* * * * * * * * * * * * * * * * * * * * * * * * * * * * * * * * * * * * * * * * * * * * * * * * * * *)$

$* * * * * * * * * * * * * * * * * * * * * * * * * * * * * *) C * * * * *$

PEP6 $* * * * * * * * * * * * * * * * *$

REP3 TGGTAACCCCGTTTGCTAATAGCACAACCAATTGAAGCTGGTATTTGGTGGCTCTGGTG REP4 - - - -

REP3 CAATTTATAG

REP4 *G****G***
REP3 $3 * * * * *-* * * * * * * * * * \mathrm{~T} * * * * * * * * * * * A G C A C C C$

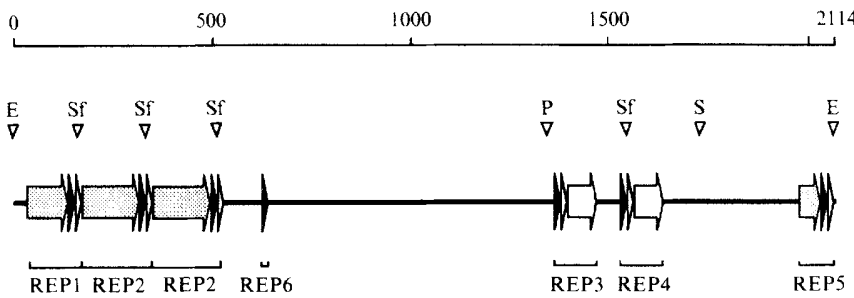

Fig. 6. Structure of RPS1. (a) Comparison of inner repeat sequences in RPS1. Positions at which the same base occurs as in REP2 or REP3 are indicated with an asterisk. Gaps between sequences are indicated with dashes. The initiation and end point, and the sizes of the REP sequences, are shown in the legend to Fig. 5. The $29 \mathrm{bp}$ common sequence, COM29, is indicated by a line. (b) Schematic structure of the repeated sequence of RPS 1 . The brackets below the figure indicate the inner repeat regions, REP1, 2, 3, 4, 5 and 6. The REP1 and REP3 families are denoted by stippled and open arrows, respectively. The filled arrowheads indicate the half of the COM29 sequence which corresponds to REP6. The open arrowheads indicate the other half of COM29. The downward-pointing open arrowheads indicate restriction sites: $E c o$ RI (E), Pst I (P), SmaI (S) and SfiI (Sf). The upper scale line shows the length of RPS1 (bp)

direct repeats of REP5, REP1, REP2 and REP2 seem to be adjacent. REP3 and REP4 are directly repeated with 67 bases of spacer sequence although REP4 has a 9 bp gap in the middle region of REP3. REP1, REP2, REP3, REP4 and REP5 all contain a 29 base common sequence, CAAAAAAGGCCGTTTTGGCCATAGTTAAG. REP6 has only 16 bases homologous with the first half of the $29 \mathrm{bp}$ common sequence. A schematic location of the repeated sequences for RPS1 is shown in Fig. 6(b).

\section{Sequence of RPSI}

The DNA sequence of RPS1 is shown in Fig. 5. RPS1 consists of $2114 \mathrm{bp}$ and its GC content is $40 \mathrm{~mol} \%$. Only one long open reading frame was found, from nucleotides 653 to 973 . However, analysis using Fickett's method did not suggest a translated protein.

In the RPS1 sequence, we found smaller repeated sequences which were named REP1 (39-172), REP2 (173-344 and 345-516), REP3 (1407-1511), REP4 (1579-1673), REP5 (2027-2108) and REP6 (662-677). REP2 is tandemly duplicated. The homology between these repeats is compared in Fig. 6(a). REP1 and REP2 are tandemly repeated; however, 36 bases of the $5^{\prime}$ region of REP2 are not homologous. The homologous regions are $95 \%$ identical. REP5 is also homologous to REP2 but 90 bases of the $5^{\prime}$ region are missing, and the rest of the region is less homologous ( $81 \%$ identical) compared to REP1. Assuming RPS1 is tandemly repeated, the $3^{\prime}$ end of the RPS1 repeat unit adjoins the next $5^{\prime}$ end. Therefore, REP5 and REP1 are directly repeated with about 39 bases of spacer sequence. Accordingly, the four

\section{Discussion}

A repeated sequence, RPS1, approximately $2 \mathrm{~kb}$ in size, was found primarily on chromosome 6 , which is the second most variable of the eight chromosomes of $C$. albicans. The most variable chromosome, chromosome 2, carries rDNA which is only located on chromosome 2 , in a tandemly repeated fashion. The rDNA unit size is estimated to be $15 \mathrm{~kb}$. The number of rDNA units varied from about 50 to about 130 per cell among the strains tested in our previous study (Iwaguchi et al., 1992). The number of RPS1 units is about 80 per cell, but the number may be very variable between strains because intensity of the hybridization signal varies between strains; for example it was extremely weak in NUM1000 (Fig. 1b, lane 4). RPS1 or homologous sequences were present not only on chromosome 6 but also on all other chromosomes except chromosome 4.

Most of the chromosome 6 RPS1 units seem to be 
located within a very limited region of about $100 \mathrm{~kb}$ in strain FC18. At least in strains FC18 and NUM812, a part of RPS1 is inferred to be tandemly repeated from the evidence that the same-sized fragments were detected by digestion with various enzymes (Fig. 3). More direct evidence was obtained by a partial SmaI digestion of chromosome 6 from strain FC18. A ladder of bands corresponding to multiple copies of RPS1 was detected by Southern hybridization and the ladder number was at least 10 (Fig. 4). This suggested that more than 10 RPS1 units are tandemly repeated in chromosome 6 .

RPS1 contains small repeat units, about $80-170$ bp in size, which we have called REP1, REP2, REP3, REP4 and REP5; REP2 is duplicated. The REPs are classified into two groups by their homology (Figs 5 and 6). One, the REP1 family, contains REP1, REP2 and REP5; the other, the REP 3 family, contains REP 3 and REP4. Both families contain a common $29 \mathrm{bp}$ sequence, called COM29. This $29 \mathrm{bp}$ sequence resembles a specific recombination site such as the $\lambda$ attachment site and the site of DNA inversion/crossing-over in bacteria (Ehrlich, 1989). For example, the site of bacterial DNA inversion is a $26 \mathrm{bp}$ sequence, composed of a $12 \mathrm{bp}$ imperfect palindrome that consists of a GC-pair-rich core and an AT-pair-rich outer region. The first part of COM 29 is composed of a $16 \mathrm{bp}$ imperfect palindrome which resembles the bacterial DNA inversion sequence. The $16 \mathrm{bp}$ palindrome of COM29, REP6, is present away from the other REPs. COM29 might be a candidate for a specific recombination site in C. albicans. REP2 might have been duplicated very recently because the copies are identical and duplication has occurred in the sequences flanked by COM29. This may support the notion that COM29 is involved in recombination events.

It is noteworthy that the COM29 region of REP1, REP 2 and REP4 contains a cutting site for $S f i \mathrm{I}$, which is an 8-base recognition restriction enzyme and useful for genome analysis. Genome mapping of $C$. albicans is being carried out by using $S f i \mathrm{I}$ (P. T. Magee, personal communication).

Dispersed repetitive elements have been characterized in chromosomes of various organisms and are causal agents of chromosomal rearrangements such as deletions, duplications, inversions and translocations (Petes \& Hill, 1988; Shapiro, 1983). The S. cerevisiae genome contains three repetitive elements, delta, tau and sigma, which are several hundred base pairs long. The delta and sigma elements were identified as the transposon terminal repeats, Ty elements. Tau is thought to be a similar element because of the significant regions of homology with the delta and sigma sequences (Genbauffe et al., 1984). In C. albicans, transposon-like elements have not been identified yet. Several repeated sequences have been isolated in C. albicans: $27 \mathrm{~A}$ of $15 \mathrm{~kb}$
(Scherer \& Stevens, 1988), Ca3 of $12 \mathrm{~kb}$ (Sadhu et al., 1991), Ca7 of $9.5 \mathrm{~kb}$ (Soll et al., 1987), the MspI fragment of $2.9 \mathrm{~kb}$ (Cutler et al., 1988), Care-1 of $0.47 \mathrm{~kb}$ (Lasker et al., 1991), and Care-2 of $1 \cdot 1 \mathrm{~kb}$ (GenBank, M74014). Ca7 has been shown to be a telomeric or subtelomeric repeat (Sadhu et al., 1991). Only Care-1 and Care-2 have been sequenced; the sequences are not homologous to the RPS1 sequence or to each other. RPS1 strongly hybridized to 27A and $\mathrm{Ca} 3$ but not to the $M s p \mathrm{I}$ fragment or Ca7 (data not shown). Thus RPS1 seems to contain a sequence of the same repeat family as the sequence contained in 27A and $\mathrm{Ca} 3$. A detailed comparison of RPS 1 and $27 \mathrm{~A}$ or $\mathrm{Ca} 3$ is in progress.

We failed to find significant homology with any known sequence within the GenBank or EMBL databases. At present, the origin of RPS1 is unknown.

We thank T. J. Lott (Centers for Disease Control, USA) for critically reading the manuscript, and D. R. Soll (University of Iowa, USA), S. Scherer (University of Minnesota, USA) and J. E. Cutler (Montana State University, USA) for gifts of the phages and the plasmids containing $C$. albicans repeat sequence.

\section{References}

Asakura, K., Iwaguchi, S.-I., Homma, M., Sukai, T., Higashide, K. \& TANAKA, K. (1991). Electrophoretic karyotypes of clinically isolated yeasts of Candida albicans and C. glabrata. Journal of General Microbiology 137, 2531-2538.

Chu, G., Vollrath, D. \& Davis, R. W. (1986). Separation of large DNA molecules by contour-clamped homogeneous electric fields. Science 234, 1582-1585.

Cutler, J. E., Glee, P. M. \& Horn, H. L. (1988). Candida albicansand Candida stellatoidea-specific DNA fragment. Journal of Clinical Microbiology 26, 1720-1724.

EHRLICH, S. D. (1989). Illegitimate recombination in bacteria. In Mobile DNA, pp. 799-832. Edited by D. E. Berg \& M. M. Howe. Washington, DC: American Society for Microbiology.

Fan, J.-B., Chikashige, Y., Smith, C. L., Niwa, O., Yanagida, M. \& CANTOR, C. R. (1988). Construction of a NotI restriction map of the fission yeast Schizosaccharomyces pombe genome. Nucleic Acids Research 17, 2801-2818.

Genbauffe, F. S., Chisholm, G. E. \& Cooper, T. G. (1984). Tau, sigma, and delta: a family of repeated elements in yeast. Journal of Biological Chemistry 259, 10518-10525.

IwaGuChI, S.-I., Homma, M. \& TanaKa, K. (1990). Variation in the electrophoretic karyotype analysed by the assignment of DNA probes in Candida albicans. Journal of General Microbiology 136, $2433-2442$.

IWAGUCHI, S.-I., Homma, M. \& TANAKA, K. (1992). Clonal variation of chromosome size derived from the rDNA cluster region in Candida albicans. Journal of General Microbiology 138, 1177-1184.

KIRSCH, D. R., LaI, M. H. \& O'Sullivan, J. (1988). Isolation of the gene for cytochrome P450L1A1 (lanosterol 14 $\alpha$-demethylase) from Candida albicans. Gene 68, 229-237.

Lasker, B. A., Carle, G. F., Kobayashi, G. S. \& Medoff, G. (1989). Comparison of the separation of Candida albicans chromosome-sized DNA by pulsed-field gel electrophoresis techniques. Nucleic Acids Research 17, 3783-3793.

Lasker, B. A., Page, L. S., Lott, T. J., Kobayashi, G. S. \& Medoff, G. (1991). Characterization of CARE-1: Candida albicans repetitive element-1. Gene 102, 45-50.

LoTT, T. J., BoIRoN, P. \& ReISs, E. (1987). An electrophoretic 
karyotype for Candida albicans reveals large chromosomes in multiples. Molecular and General Genetics 209, 170-174.

MAGEE, B. B. \& MAGEE, P. T. (1987). Electrophoretic karyotypes and chromosome numbers in Candida species. Journal of General Microbiology 133, 425-430.

Merz, W. G., Connelly, C. \& Hieter, P. (1988). Variation of electrophoretic karyotypes among clinical isolates of Candida albicans. Journal of Clinical Microbiology 26, 842-845.

MORTIMER, R. K. \& SCHILD, D. (1985). Genetic map of Saccharomyces cerevisiae, edition 9. Microbiological Reviews 49, 181-212.

Petes, T. D. \& Hill, C. W. (1988). Recombination between repeated genes in microorganisms. Annual Review of Genetics 22, 147-168.

RustChenKo-BulgaC, E. P. (1991). Variations of Candida albicans electrophoretic karyotypes. Journal of Bacteriology 173, 6586-6596.

SAdHu, C., McEachern, M. J., Rustchenko-Bulgac, E. P., Schmid, J., SolL, D. R. \& HICKs, J. B. (1991). Telomeric and dispersed repeat sequences in Candida yeasts and their use in strain identification. Journal of Bacterioloy 173, 842-850.

SCHERER, S. \& MAGEE, P. T. (1990). Genetics of Candida albicans. Microbiological Reviews 54, 226-241.
Scherer, S. \& Stevens, D. A. (1988). A Candida albicans dispersed, repeated gene family and its epidemiologic applications. Proceedings of the National Academy of Sciences of the United States of America 85, $1452-1456$.

ShapIRo, J. A. (1983). Mobile Genetic Elements. New York: Academic Press.

SNell, R. G., Hermans, I. F., Wilkins, R. J. \& Corner, B. E. (1987) Chromosomal variation in Candida albicans. Nucleic Acids Research 15, 3625 .

Soll, D. R., Langtimm, C. J., McDowell, J., Hicks, J. \& Galask, R. (1987). High-frequency switching in Candida strains isolated from vaginitis patients. Journal of Clinical Microbiology 25, 1611-1622.

Szostak, J. W. \& WU, R. (1980). Unequal crossing over in the ribosomal DNA of Saccharomyces cerevisiae. Nature, London 284 426-430.

Wickes, B., Staudinger, J., Magee, B. B., Kwon-Chung, K.-J. MAGEe, P. T. \& SCHERER, S. (1991). Physical and genetic mapping of Candida albicans, several genes previously assigned to chromosome 1 map to chromosome $\mathrm{R}$, the rDNA-containing linkage group. Infection and Immunity 59, 2480-2484. 